\title{
On inertial navigation and attitude initialization in polar areas
}

\author{
Yuanxin $\mathrm{Wu}^{1 *} \mathbb{0}$, Chao $\mathrm{He}^{2}$ and Gang Liu²
}

\begin{abstract}
Inertial navigation and attitude initialization in polar areas become a hot topic in recent years in the navigation community, as the widely-used navigation mechanization of the local level frame encounters the inherent singularity when the latitude approaches $90^{\circ}$. Great endeavors have been devoted to devising novel navigation mechanizations such as the grid or transversal frames. This paper highlights the fact that the common Earth-frame mechanization is sufficiently good to handle the singularity problem in polar areas. Simulation results are reported to demonstrate the singularity problem and the effectiveness of the Earth-frame mechanization.
\end{abstract}

Keywords: Inertial navigation, Mechanization, Polar areas, Attitude initialization

\section{Introduction}

There are increasing demands of human activities in polar areas, such as civil aviation and underwater resource exploration. Strapdown inertial navigation systems are a kind of standard equipment for airplanes and submarines to fulfill autonomous and reliable navigation in performing those activities.

Polar applications have raised a couple of challenges for inertial navigation systems [1]. One is the problem of attitude initialization or alignment, as the Earth gravity and the Earth rotation vector turn to be parallel near two poles, which poses a big trouble in determining the initial attitude condition for the self-contained inertial navigation systems to start with. In this regard, aiding information is indispensable to help initialize inertial navigation systems, for instance by the global navigation satellite system (GNSS). Fortunately, GNSS is able to provide reliable position and velocity information if an accurate troposphere delay model combined with dual-frequency ionosphere cancellation is used to overcome the problems caused by low satellite elevation [2]. The other problem is related to the widely-used computation

\footnotetext{
*Correspondence: yuanx_wu@hotmail.com

1 Shanghai Key Laboratory of Navigation and Location-based Services, School of Electronic Information and Electrical Engineering, Shanghai Jiao Tong University, Shanghai 200240, China

Full list of author information is available at the end of the article
}

mechanization of the (north-pointing) local-level frame, because the north or south directions vary fast along with movement towards high-attitude regions. A partial remedy is to use the wander-azimuth local-level frame yet at the expense of the north direction and longitude outputs near the poles [1]. The recent years have witnessed a number of works on grid or transversal frames based mechanizations, see e.g. [3-8], endeavoring to find a globally-deployable navigation mechanization to surmount the above polar singularity. However, the proposed grid or transversal navigation mechanizations have to involve to-and-fro transformations and even mechanization switches at lower-latitude areas, significantly complicating the navigation computer tasks. In fact, the current paper argues that many practitioners have neglected an obvious fact that the common Earth frame could be simply used for inertial navigation systems to achieve the global navigation ability. The work [7] actually employs this fact yet using a concept of normal vector to encode the curvilinear position information.

\section{Attitude alignment and navigation computation in Earth frame}

Throughout the paper, the WGS-84 (World Geodetic System) is used. In the Earth-centered Earth-fixed (ECEF) frame, the inertial navigation (attitude, velocity and position) rate equations are well-known as [1] 


$$
\begin{aligned}
& \dot{\mathbf{C}}_{b}^{e}=\mathbf{C}_{b}^{e}\left(\boldsymbol{\omega}_{e b}^{b} \times\right) \\
& \dot{\mathbf{v}}^{e}=\mathbf{C}_{b}^{e} \mathbf{f}^{b}-2 \boldsymbol{\omega}_{i e}^{e} \times \mathbf{v}^{e}+\mathbf{g}^{e} \\
& \dot{\mathbf{p}}^{e}=\mathbf{v}^{e}
\end{aligned}
$$

where $\mathbf{p}^{e}=\left[\begin{array}{lll}x & y & z\end{array}\right]^{T}$ denotes the ECEF coordinate of the vehicle, $\mathbf{v}^{e}=\left[\begin{array}{lll}v_{x} & v_{y} & v_{z}\end{array}\right]^{T}$ is the ground velocity expressed in the Earth frame and $\omega_{i e}^{e}=\left[\begin{array}{lll}0 & 0 & \Omega\end{array}\right]^{T}$ is the Earth rotation rate expressed in the Earth frame. $\Omega$ is the Earth rotation rate. $\mathbf{C}_{e}^{b}$ denotes the body attitude matrix from the Earth frame to the body frame, $\boldsymbol{\omega}_{e b}^{b}=\boldsymbol{\omega}_{i b}^{b}-\mathbf{C}_{e}^{b} \boldsymbol{\omega}_{i e}^{e}$ the body angular rate with respect to the Earth frame, $\boldsymbol{\omega}_{i b}^{b}$ the body angular rate measured by gyroscopes in the body frame, $\mathbf{f}^{b}$ the specific force measured by accelerometers in the body frame, and $\mathbf{g}^{e}$ the gravity vector. The $3 \times 3$ skew symmetric matrix $(\cdot \times)$ is defined so that the cross product satisfies $\mathbf{a} \times \mathbf{b}=(\mathbf{a} \times) \mathbf{b}$ for arbitrary two vectors.

\section{Attitude alignment}

In general, inertial navigation systems cannot effectively accomplish attitude alignment by themselves in polar areas, as the gravity and Earth rotation vectors turn to be on the same line. In order to be initialized quickly and accurately, an aid of GNSS for aviation or a doppler velocity logger for underwater vehicles is necessary [9, 10]. The fine alignment stage usually relies on the practical technique of extended Kalman filtering for accurate attitude as well as online calibration of inertial sensors. Again, a good initial attitude state is vital for the Kalman filtering to behave reliably. The optimization-based alignment method [10-12] has been widely accepted by the community to produce a coarse but good enough initial attitude for the subsequent Kalman-filtering based fine alignment. Hereby we briefly review it in the context of Earth frame and explain how the velocity maneuvers could help speed up the GNSS-aided attitude alignment in polar applications. In reference to the work [10], the coarse attitude alignment in the Earth frame could be formulated as

$$
\mathbf{C}_{b}^{e}(0) \boldsymbol{\alpha}=\boldsymbol{\beta}
$$

where

$$
\begin{aligned}
& \boldsymbol{\alpha} \triangleq \int_{0}^{t} \mathbf{C}_{b(t)}^{b(0)} \mathbf{f}^{b} d t \\
& \boldsymbol{\beta} \triangleq \mathbf{C}_{e(t)}^{e(0)} \mathbf{v}^{e}-\mathbf{v}^{e}(0)+\int_{0}^{t} \mathbf{C}_{e(t)}^{e(0)} \boldsymbol{\omega}_{i e}^{e} \times \mathbf{v}^{e} d t-\int_{0}^{t} \mathbf{C}_{e(t)}^{e(0)} \mathbf{g}^{e} d t
\end{aligned}
$$

The initial attitude matrix at time zero, namely $\mathbf{C}_{b}^{e}(0)$, can be determined by solving an eigenvector/eigenvalue problem, if the vector $\alpha$ or $\beta$ changes its direction in the time interval of interest. In the perspective of numerical computation, more significant the vector direction changes, more accurate the attitude matrix will be. Then the coarse attitude matrix at current time is to be obtained by $\mathbf{C}_{b}^{e}(t)=\mathbf{C}_{e(0)}^{e(t)} \mathbf{C}_{b}^{e}(0) \mathbf{C}_{b(t)}^{b(0)}$. A closer look tells that the vector $\alpha$ solely depends on the outputs of inertial navigation systems and the vector $\beta$ is determined by the GNSS position and velocity as well as the inertially apparent gravity $\mathbf{C}_{e(t)}^{e(0)} \mathbf{g}^{e}$. In polar areas, the apparent gravity roughly concentrates on a line and contributes little to the direction change of the vector $\beta$. However, this shortcoming could be effectively overcome by the vehicle's velocity maneuvers, especially the direction-varying velocity maneuvers.

In principle, attitude alignment in the Earth frame has no big difference from that in the local-level frame, except free of polar singularity. For details, readers are referred to Refs. [10, 12].

\section{Navigation computation}

The navigation computation procedure in the Earth frame roughly follows that of the local-level frame (North-UpEast), except being free of the singularity. Table 1 lists and compares the typical two-sample computation procedures in the Earth frame and the local-level frame [1]. Without loss of generality, the navigation computation is considered in the time interval $\left[t_{k} t_{k+1}\right]$, which contains two samples of gyroscopes (denoted as incremental angles $\Delta \boldsymbol{\theta}_{1}, \Delta \boldsymbol{\theta}_{2}$ ) and accelerometers (denoted as incremental velocities $\Delta \mathbf{v}_{1}, \Delta \mathbf{v}_{2}$ ) and $t_{k+1}-t_{k} \triangleq T$. The local curvature matrix $\mathbf{R}_{c}$ is a function of the current position (longitude $\lambda$, latitude $L$ and height $h$ ) as

$$
\mathbf{R}_{c}=\left[\begin{array}{ccc}
0 & 0 & \frac{1}{\left(R_{E}+h\right) \cos L} \\
\frac{1}{R_{N}+h} & 0 & 0 \\
0 & 1 & 0
\end{array}\right]
$$

where $R_{E}$ and $R_{N}$ are respectively the transverse radius of curvature and the meridian radius of curvature of the reference ellipsoid, depending on the current position as well. Specifically, the local curvature matrix $\mathbf{R}_{c}$ and the navigation frame's angular rate $\omega_{e n}^{n}$ will be subject to singularity problems while the latitude $L$ approaches $90^{\circ}$. This is why the local-level mechanization cannot work properly near two poles. Note that throughout the paper the attitude matrix $\mathbf{C}_{x}^{y}$ is related to the attitude quaternion $\mathbf{q}_{y}^{x} \triangleq\left[\begin{array}{ll}s & \eta^{T}\end{array}\right]^{T}$ by

$$
\mathbf{C}_{x}^{y}=\left(s^{2}-\eta^{T} \boldsymbol{\eta}\right) \mathbf{I}_{3}+2 \boldsymbol{\eta} \boldsymbol{\eta}^{T}+2 s \boldsymbol{\eta} \times
$$

It may be argued that the navigation information in the Earth frame is not intuitive for human operators to 
Table 1 Two-sample inertial navigation computation in local-level frame and Earth frame

\begin{tabular}{|c|c|c|}
\hline & Local-level frame & Earth frame \\
\hline \multirow[t]{6}{*}{ Attitude update } & $\boldsymbol{\omega}_{i e}^{n}=[\Omega \cos L \Omega \sin L 0]^{\top}$ & $\boldsymbol{\omega}_{i e}^{e}=\left[\begin{array}{lll}0 & 0 & \Omega\end{array}\right]^{T}$ \\
\hline & $\boldsymbol{\omega}_{e n}^{n}=\left[v_{E}^{n} /\left(R_{E}+h\right) v_{E}^{n} \tan L /\left(R_{E}+h\right)-v_{N}^{n} /\left(R_{N}+h\right)\right]^{T}$ & $\boldsymbol{\sigma}_{e}=T \boldsymbol{\omega}_{i e}^{e}$ \\
\hline & $\boldsymbol{\sigma}_{n}=T\left(\boldsymbol{\omega}_{i e}^{n}+\boldsymbol{\omega}_{e n}^{n}\right)$ & $\boldsymbol{q}_{e}^{e_{k+1}}=\cos \frac{\left|\boldsymbol{\sigma}_{e}\right|}{e}+\frac{\boldsymbol{\sigma}_{e}}{\sin } \operatorname{si} \frac{\left|\boldsymbol{\sigma}_{e}\right|}{2}$ \\
\hline & $\mathbf{q}_{n_{k}}^{n_{k+1}}=\cos \frac{\left|\boldsymbol{\sigma}_{n}\right|}{2}+\frac{\boldsymbol{\sigma}_{n}}{\left|\boldsymbol{\sigma}_{n}\right|} \sin \frac{\left|\boldsymbol{\sigma}_{n}\right|}{2}$ & $\mathbf{4}_{k}-\operatorname{los} \frac{\left|\boldsymbol{\sigma}_{e}\right|}{2}$ \\
\hline & $\boldsymbol{\sigma}_{b}=\Delta \boldsymbol{\theta}_{1}+\Delta \boldsymbol{\theta}_{2}+\frac{2}{3} \Delta \boldsymbol{\theta}_{1} \times \Delta \boldsymbol{\theta}_{2}, \quad \mathbf{q}_{b_{k}}^{b_{k+1}}=\cos \frac{\left|\boldsymbol{\sigma}_{b}\right|}{2}+\frac{\boldsymbol{\sigma}_{b}}{\left|\boldsymbol{\sigma}_{b}\right|} \sin \frac{\left|\boldsymbol{\sigma}_{b}\right|}{2}$ & \\
\hline & $\mathbf{C}_{b}^{n}(k+1)=\mathbf{C}_{n_{k}}^{n_{k+1}} \mathbf{C}_{b}^{n}(k) \mathbf{C}_{b_{k+1}}^{b_{k}}$ & $\mathbf{C}_{b}^{e}(k+1)=\mathbf{C}_{e_{k}}^{e_{k+1}} \mathbf{C}_{b}^{e}(k) \mathbf{C}_{b_{k+1}}^{b_{k}}$ \\
\hline \multirow[t]{3}{*}{ Velocity update } & $\mathbf{u}^{b}=\Delta \mathbf{v}_{1}+\Delta \mathbf{v}_{2}+\frac{1}{2}\left(\Delta \boldsymbol{\theta}_{1}+\Delta \boldsymbol{\theta}_{2}\right) \times\left(\Delta \mathbf{v}_{1}+\Delta \mathbf{v}_{2}\right)+\frac{2}{3}\left(\Delta \boldsymbol{\theta}_{1} \times \Delta \mathbf{v}_{2}+\Delta \mathbf{v}_{1}\right.$ & $\times \Delta \boldsymbol{\theta}_{2}$ \\
\hline & $\mathbf{v}^{n}(k+1)=\mathbf{v}^{n}(k)+\mathbf{C}_{b}^{n}(k) \mathbf{u}^{b}$ & $\mathbf{v}^{e}(k+1)=\mathbf{v}^{e}(k)+\mathbf{C}_{b}^{e}(k) \mathbf{u}^{b}$ \\
\hline & $-T\left(2 \boldsymbol{\omega}_{i e}^{n}+\boldsymbol{\omega}_{e n}^{n}\right) \times \mathbf{v}^{n}(k)+T \mathbf{g}^{n}(k)$ & $-2 T \boldsymbol{\omega}_{i e}^{e} \times \mathbf{v}^{e}(k)+T \mathbf{g}^{e}(k)$ \\
\hline \multirow[t]{2}{*}{ Position update } & $\mathbf{r}=T\left(\mathbf{v}^{n}(k)+\mathbf{v}^{n}(k+1)\right) / 2$ & $\mathbf{p}^{e}(k+1)=\mathbf{p}^{e}(k)+T\left(\mathbf{v}^{e}(k)+\mathbf{v}^{e}(k+1)\right) / 2$ \\
\hline & $\mathbf{p}^{n}(k+1)=\mathbf{p}^{n}(k)+\mathbf{R}_{c}(k) \mathbf{r}$ & \\
\hline
\end{tabular}

comprehend or act accordingly. As a matter of fact, this concern could be readily spared by simple coordinate transformation out of the navigation computation procedure, e.g., from the ECEF coordinate to its counterpart in the local-level frame. Figure 1 presents the flowchart of the navigation mechanization in the Earth frame, including the possible coordinate transformation for intuitive display.

\section{Numerical results of polar navigation}

This section simulates and compares the performance of the local-level and Earth-frame mechanizations in polar areas. Without loss of generality, perfect sensors (no sensor error considered) and a sphere globe are considered, which does not alter the conclusions to be obtained hereafter.
Two flight scenarios have been considered, in which the flights both start from the location of longitude $120^{\circ}$ and latitude $50^{\circ}$, a location near the Chinese city of Qiqihar. The height is kept constant at $10,000 \mathrm{~m}$ and the speed is also kept constant at $2000 \mathrm{~m} / \mathrm{s}$. Throughout the flight, the aircraft attitude is perfectly aligned with the Earth frame, namely $\mathbf{C}_{b}^{e}=\mathbf{I}_{3}$.

In the first scenario, the aircraft flies southward for an hour, arriving at latitude about minus $15^{\circ}$ in the south hemisphere, while in the second scenario, it flies northward for $1 \mathrm{~h}$ and a half, passing the north pole and finally arriving at the location of longitude $60^{\circ}$ and latitude about $33^{\circ}$. The two flight paths are demonstrated in Fig. 2.

The simulation data are generated analytically. Specifically, the aircraft positions in both the curvilinear and ECEF coordinates are given by

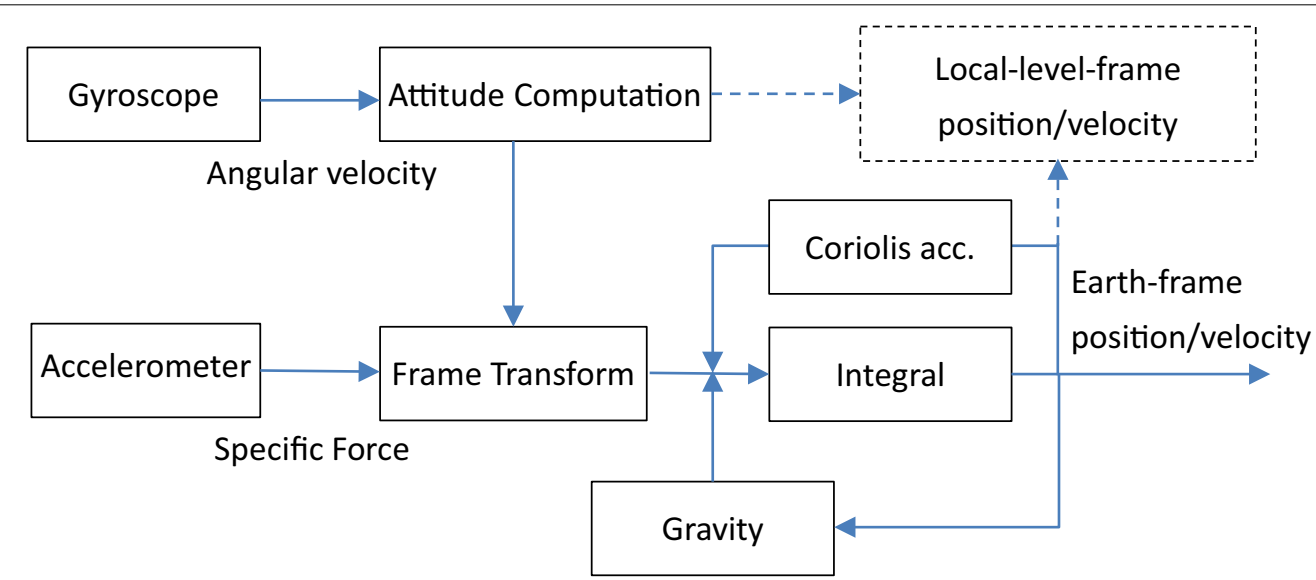

Fig. 1 Earth-frame inertial navigation mechanization (with possible information display in local-level frame, as indicated by dashed lines and rectangular) 

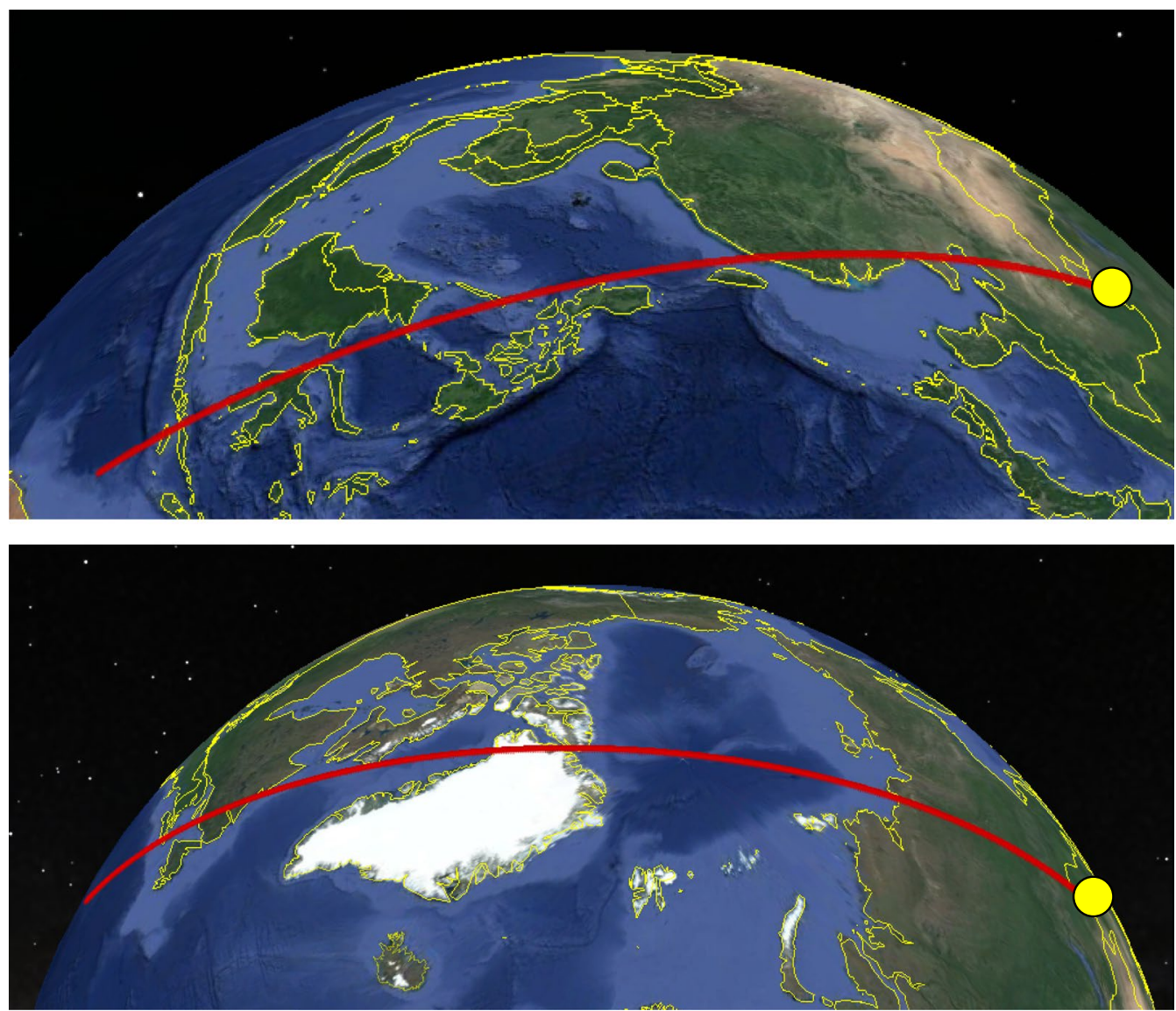

Fig. 2 Two flight scenarios. (Up) southward flight; (below) northward flight passing north pole. Yellow circles indicate starting location

$$
\begin{aligned}
& \mathbf{p}^{n} \equiv\left[\begin{array}{l}
\lambda \\
L \\
h
\end{array}\right]=\left[\begin{array}{c}
\lambda_{0} \\
\left.L_{0}+\frac{v}{R+h} t\right] \\
h_{0}
\end{array}\right], \\
& \mathbf{p}^{e} \equiv\left[\begin{array}{l}
x \\
y \\
z
\end{array}\right]=(R+h)\left[\begin{array}{c}
\cos (L) \cos \lambda \\
\cos (L) \sin \lambda \\
\sin (L)
\end{array}\right]
\end{aligned}
$$

where the initial position has $\lambda_{0}=120^{\circ}, L_{0}=50^{\circ}$ and $h_{0}=10,000 \mathrm{~m}$, and $v=2000 \mathrm{~m} / \mathrm{s}$. According to Eqs. (3) and (8), the velocity in the Earth frame and its derivative are obtained as

$$
\begin{aligned}
& \mathbf{v}^{e}=\dot{\mathbf{p}}^{e}=\left[\begin{array}{c}
-\sin (L) \cos \lambda \\
-\sin (L) \sin \lambda \\
\cos (L)
\end{array}\right] v, \\
& \dot{\mathbf{v}}^{e}=-\left[\begin{array}{c}
\cos (L) \cos \lambda \\
\cos (L) \sin \lambda \\
\sin (L)
\end{array}\right] \frac{v^{2}}{R+h}
\end{aligned}
$$

Recalling $\mathbf{C}_{b}^{e}=\mathbf{I}_{3}$ and substituting into Eq. (2) produce the specific force measured by accelerometers $\mathbf{f}^{b}=\dot{\mathbf{v}}^{e}+2 \boldsymbol{\omega}_{i e}^{e} \times \mathbf{v}^{e}-\mathbf{g}^{e}$. And then according to Eq. (1), the aircraft inertial angular velocity measured by gyroscopes is $\omega_{i b}^{b}=\omega_{i e^{e}}^{e}$

As the vertical channel is unstable, zero vertical velocity is assumed a priori known and used to reset the computation procedure after each update. As far as the Earth-frame mechanization is concerned, the velocity is first transformed to the local level frame and then back to the Earth frame after applying the zero vertical velocity reset.

The computation errors in the first scenario are plotted in Fig. 3. It shows that the two mechanizations perform comparably well in the non-polar regions, with a positioning error of about 20-60 $\mathrm{m}$. This error amount is negligible compared with the aviation-grade inertial navigation systems typically with a positioning error of a couple of kilometers per hour.

Figure 4 plots the horizontal position errors in polar navigation in the second scenario. Figure 5 presents the west-east position error versus the true latitude of the flight trajectory for the local-level mechanization. As predicated, the local-level mechanization utterly fails with its positioning error roaring to hundreds of 

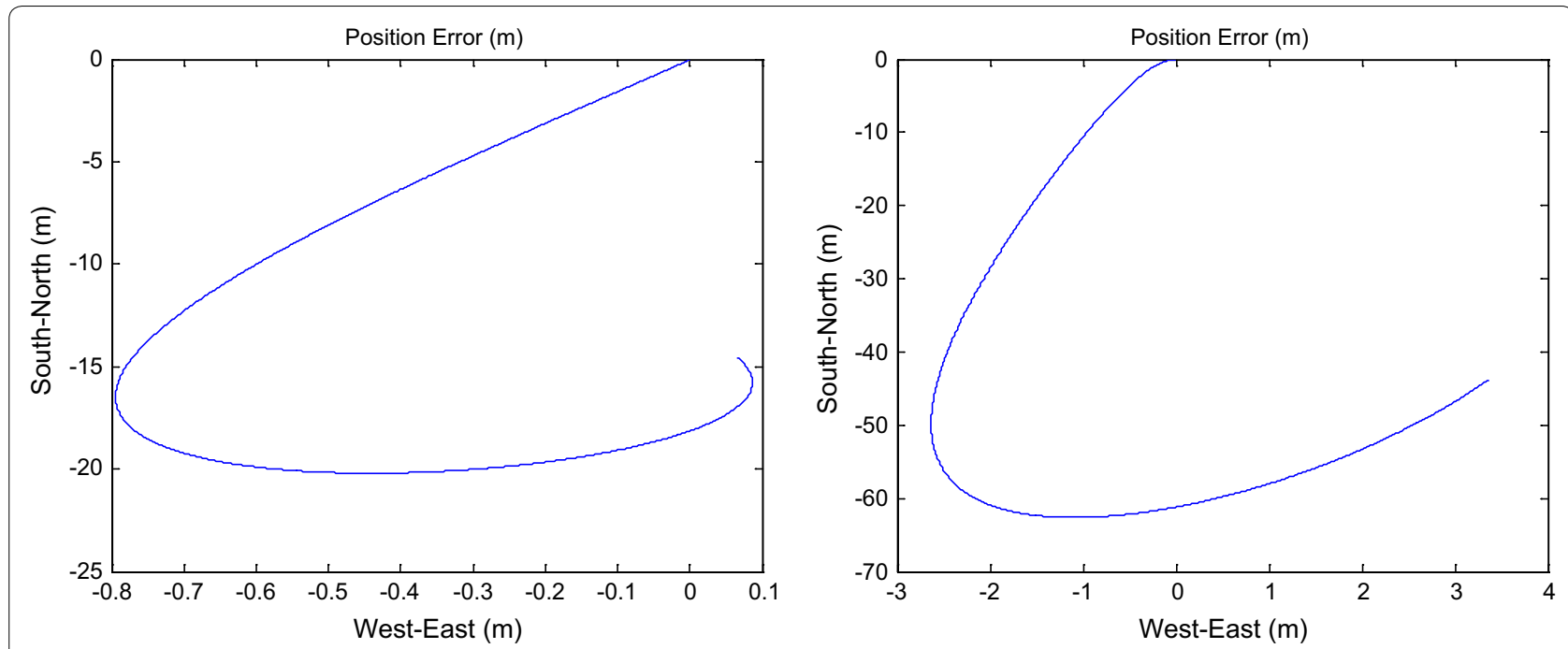

Fig. 3 Non-polar position errors of local-level-frame (left) and Earth-frame mechanizations (right)

kilometers when approaching the north pole. Meanwhile, the Earth-frame mechanization behaves in the polar region as normally as in the first scenario. The positioning error is no more than $60 \mathrm{~m}$ in the whole flight.

Though simple, the above simulation comparison is a strong support for the Earth-frame inertial navigation mechanization in various global applications.

\section{Conclusions}

Inertial navigation systems using the widely-used locallevel-frame mechanization would encounter the inherent singularity problem near two poles. This paper proposes the usual but seldomly-used Earth-frame mechanization as an alternate for polar applications. Simulation results are reported in favor of this recommendation. By way of analysis, the attitude initialization with the aid of GNSS for aviation or a doppler velocity logger for underwater applications could also be safely performed in the Earth frame, even in polar regions.
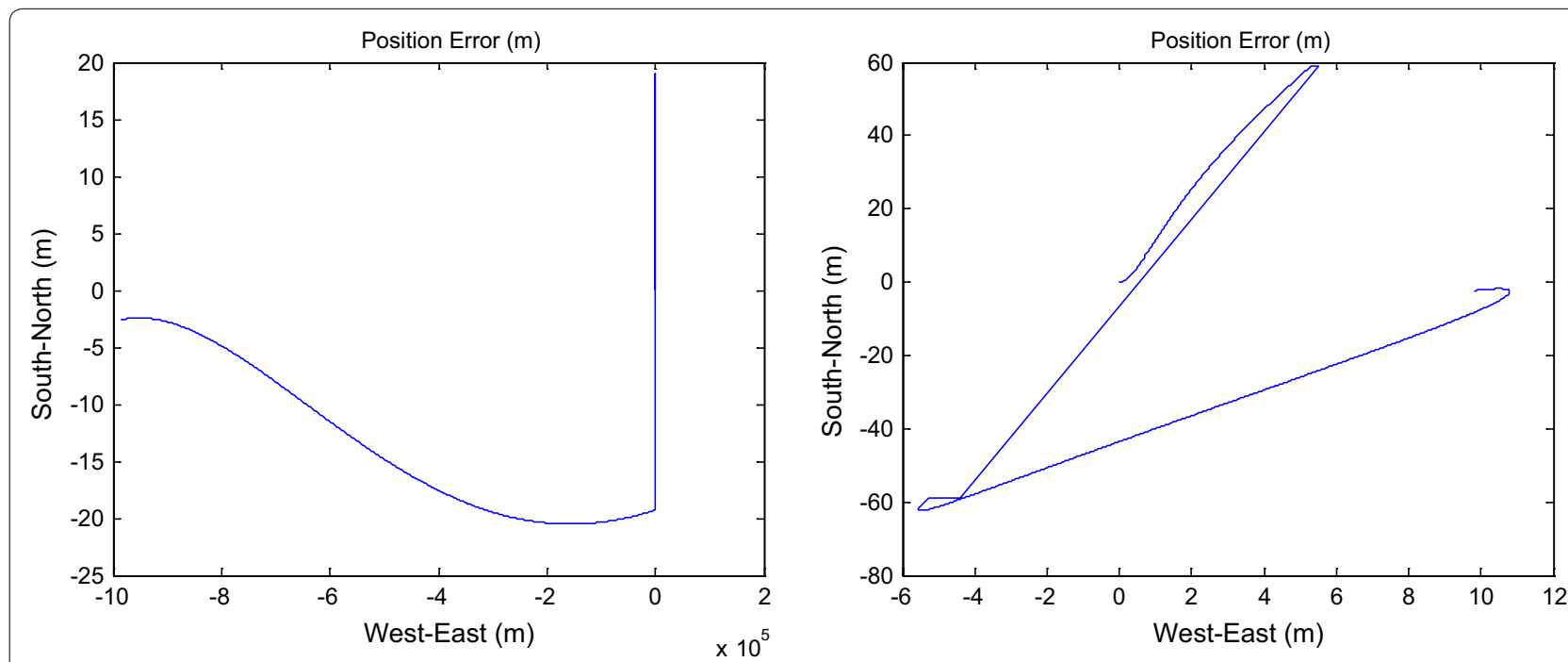

Fig. 4 Polar position errors of local-level-frame (left) and Earth-frame mechanizations (right) 


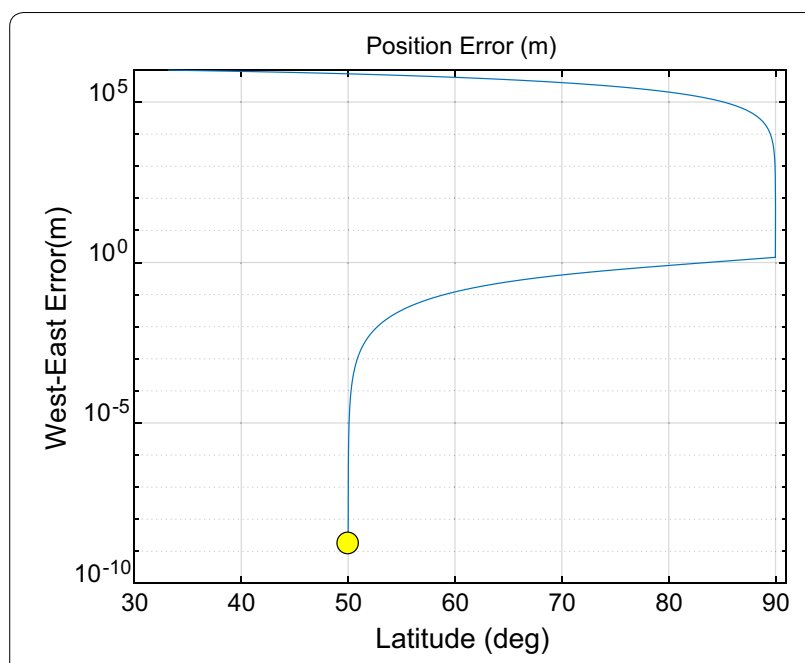

Fig. 5 Absolute west-east position errors versus true latitude of flight trajectory in local-level-frame mechanization

\section{Acknowledgements}

Not applicable.

\section{Authors' contributions}

YW proposed the idea and carried out the simulation; $\mathrm{CH}$ recommended the polar alignment part and assisted in carrying out the simulation; GL assisted in carrying out the simulation. All authors read and approved the final manuscript.

\section{Funding}

Not applicable.

\section{Availability of data and materials}

Not applicable.

\section{Competing interests}

The authors declare that they have no competing interests.

\section{Author details}

${ }^{1}$ Shanghai Key Laboratory of Navigation and Location-based Services, School of Electronic Information and Electrical Engineering, Shanghai Jiao Tong
University, Shanghai 200240, China. ${ }^{2}$ Flight Automatic Control Research Institute, Xi'an 710065, China.

Received: 29 May 2019 Accepted: 30 September 2019

Published online: 20 January 2020

\section{References}

1. Groves, P. D. (2013). Principles of GNSS, inertial, and multisensor integrated navigation systems. Boston: Artech House.

2. Yang, Y., \& Xu, J. (2016). Positioning performance analysis of Beidou in polar areas. Geomatics and Information Science of Wuhan University, 41(1), 15-20.

3. Li, Q., Ben, Y., Yu, F., \& Sun, F. (2015). System reset of transversal strapdown INS for ship in polar region. Measurement, 60, 247-257.

4. Li, Q., Ben, Y., Yu, F., \& Tan, J. (2016). Transversal strapdown INS based on reference ellipsoid for vehicle in the polar region. IEEE Transactions on Vehicular Technology, 65(9), 7791-7795.

5. Li, Q., Sun, F., Ben, Y.-Y., \& Yu, F. (2014). Polar navigation of strapdown inertial navigation system based on transversal frame in polar region. Journal of Chinese Inertial Technology, 22(3), 288-295.

6. Qin, F., Chang, L., \& Li, A. (2018). Improved transversal polar navigation mechanism for strapdown INS using ellipsoidal Earth model. Journal of Navigation, 71(6), 1460-1476.

7. Zhang, G., Yan, G., Weng, J., \& Yang, H. (2017). N-vector inertial navigation mechanization algorithm for transpolar aircraft. Journal of Chinese Inertial Technology, 25(5), 605-610.

8. Zhou, Q., Qin, Y., Fu, Q., \& Yue, Y. (2013). Grid mechanization in inertial navigation systems for transpolar aircraft. Journal of Northwestern Polytechnical University, 31(2), 210-217.

9. Li, W., Tang, K., Lu, L., \& Wu, Y. (2013). Optimization-based INS in-motion alignment approach for underwater vehicles. Optik International Journal for Light Electron Optics, 124(20), 4581-4585.

10. Wu, Y., \& Pan, X. (2013). Velocity/position integration formula (I): Application to in-flight coarse alignment. IEEE Transactions on Aerospace and Electronic Systems, 49(2), 1006-1023.

11. Wu, M., Wu, Y., Hu, X., \& Hu, D. (2011). Optimization-based alignment for inertial navigation systems: Theory and algorithm. Aerospace Science and Technology, 15(1), 1-17.

12. Wu, Y., Wang, J., \& Hu, D. (2014). A new technique for INS/GNSS attitude and parameter estimation using online optimization. IEEE Transactions on Signal Processing, 62(10), 2642-2655.

\section{Publisher's Note}

Springer Nature remains neutral with regard to jurisdictional claims in published maps and institutional affiliations.

\section{Submit your manuscript to a SpringerOpen ${ }^{\circ}$ journal and benefit from:}

- Convenient online submission

- Rigorous peer review

- Open access: articles freely available online

- High visibility within the field

- Retaining the copyright to your article

Submit your next manuscript at $\boldsymbol{\nabla}$ springeropen.com 